\title{
MAPPING SEYFERT AND LINER EXCITATION MODES IN THE INNER KPC OF NGC 3393
}

\author{
W. Peter Maksym ${ }^{1}$, Giuseppina Fabbiano ${ }^{1}$, Martin Elvis ${ }^{1}$, Margarita Karovska ${ }^{1}$, Alessandro Paggi ${ }^{1}$, \\ John RAYMOnd ${ }^{1}$, Junfeng WANG ${ }^{2}$, AND Thaisa Storchi-BERgMann ${ }^{3}$ \\ ${ }^{1}$ Harvard-Smithsonian Center for Astrophysics, 60 Garden Street, Cambridge, MA 02138, USA; walter.maksym@cfa.harvard.edu \\ ${ }^{2}$ Department of Astronomy, Physics Building, Xiamen University, Xiamen, Fujian, 361005, China \\ ${ }^{3}$ Departamento de Astronomia, Universidade Federal do Rio Grande do Sul, IF, CP 15051, 91501-970 Porto Alegre, RS, Brazil \\ Received 2016 March 30; revised 2016 June 28; accepted 2016 July 12; published 2016 September 21
}

\begin{abstract}
We mapped the extended narrowline region (ENLR) of NGC 3393 on scales of $r \lesssim 4^{\prime \prime}(\sim 1 \mathrm{kpc})$ from the nucleus using emission line images of $\mathrm{H} \alpha \lambda 6563$, [O III] $\lambda 5007$, and [S II] $\lambda \lambda 6717,6731$, taken with the Hubble Space Telescope as part of the CHandra survey of Extended Emission line Regions in nearby Seyfert galaxies (CHEERS). By mapping these lines onto a spatially resolved Baldwin-Phillips-Terlevich diagram, we investigate the impact of feedback from a Compton-thick active galactic nucleus on its circumnuclear ISM. We find that the expected Seyfert-like emission within the ionization bicone $\left(\lesssim 3^{\prime \prime} ; 770 \mathrm{pc}\right)$. We also find a new, figure-8-shaped low ionization emission line region (LINER) cocoon enveloping the bicone and defining a sharp $(\lesssim 100 \mathrm{pc})$ transition between higher and lower-ionization zones. These data illustrate the morphological dependence of ionization states of the ENLR relative to bicone and host gas geometries.
\end{abstract}

Key words: galaxies: active - galaxies: individual (NGC 3393) - galaxies: Seyfert

\section{INTRODUCTION}

Active galactic nuclei (AGN) seem to play a critical role in the evolution of their host galaxies. As a supermassive black hole $(\mathrm{SMBH})$ accretes material, photoionizing radiation and kinetic outflows can regulate AGN accretion and galactic star formation via positive and negative feedback, which in turn affects the rate of SMBH growth (Silk \& Rees 1998; Fabian 2012; Heckman \& Best 2014). The ability to quantify the relative impact of different modes of feedback is of interest for characterizing this process, but at large distances from the observer, the inability to resolve the extended narrowline region (ENLR) limits its usefulness as a tool for characterizing AGN behavior. Detailed studies of the ENLR in nearby galaxies are therefore necessary to help understand these processes in detail (see e.g., Storchi-Bergmann et al. 2010; Wang et al. 2011a, 2011b; Paggi et al. 2012; Barbosa et al. 2014).

Low ionization emission line regions (LINERs; Heckman 1980) are a puzzling feature of AGNs and starbursts, as they may be excited by several processes (Ho 2008). Without a means to disentangle these mechanisms, these common (Nisbet \& Best 2016) objects cannot be used to cleanly investigate either AGN or starbursts.

Baldwin et al. (1981) pioneered the use of narrowline ratio diagnostic diagrams in the classification of AGNs, using ratios between a variety of lines such as $[\mathrm{N}$ II] $\lambda 6584 / \mathrm{H} \alpha \lambda 6563$ and [O III] $\lambda 5007 / \mathrm{H} \beta \lambda 4861$ to describe the observed differences between various extragalactic phenomena in terms of activity that is primarily Seyfert-driven, LINER-like, or star formation-driven (see Kewley et al. 2001, 2006). This figure is now called the "Baldwin-Phillips-Terlevich (BPT) diagram." BPT diagrams have become important tools for classifying large samples of galaxies.

It is also possible to make spatially resolved BPT diagrams such that different parts of a galaxy are mapped in terms of their BPT classification (e.g., Ho et al. 2014; Cresci et al. 2015; Davies et al. 2016, for a recent example using Integral field units).

We exploit this method in this paper to see if LINER emission has some special morphology. We use the higher, space-based, angular resolution of the Hubble Space Telescope (HST) with narrowband filters. Low-redshift galaxies present a particular opportunity due to the advantages of angular scale.

Here, we investigate the bright $\left(m_{B}=13.1\right.$; de Vaucouleurs et al. 1991) nearby face-on spiral galaxy NGC 3393 as part of CHandra survey of Extended Emission line Regions in nearby Seyfert galaxies (CHEERS; PI: J. Wang). NGC 3393 is a lowluminosity $\quad\left(L_{X}[0.5-10 \mathrm{keV}] \sim 10^{41} \mathrm{erg} \mathrm{s}^{-1}\right.$; Levenson et al. 2006) type 2 Seyfert galaxy at $z=0.0125(D=53 \mathrm{Mpc}$; Theureau et al. 1998). NGC 3393 has a water maser disk (Kondratko et al. 2008), X-rays show it is Compton-thick (Maiolino et al. 1998), and various studies imply $L_{\text {bol }} \sim 10^{44} \mathrm{erg} \mathrm{s}^{-1}$ (within an order of magnitude; see Kondratko et al. 2008; Winter et al. 2012; Baumgartner et al. 2013; Koss et al. 2015). Its host galaxy has long been known to exhibit evidence for complicated jet-NLR interactions, with a significant ENLR covering much of the galaxy, dominated by S-shaped emission line "arms" which wrap around compact sub-kpc radio jet lobes (Cooke et al. 2000).

Cooke et al. (2000) investigated the ENLR morphology using pre-COSTAR [O III] $\lambda 5007$ and $\mathrm{H} \alpha+[\mathrm{N}$ II] $\lambda \lambda 6563,6548$, and 6584 images and produced a sparsely sampled resolved BPT diagram of the entire galaxy using single-slit spectroscopy. Here, we use more recent (post-COSTAR) HST narrowband images obtained via CHEERS to investigate the ENLR excitation on $\sim 10 \mathrm{kpc}$ scales using a resolved BPT diagram and image map covering the region within $\sim 8^{\prime \prime}$ of the nucleus.

This is the first paper in a series to be followed by an indepth study of the comparative optical, X-ray and radio morphology of the NGC 3393 ENLR, as well as a study of its resolved narrowline $\mathrm{X}$-ray morphology. In this series, we describe the physical mechanisms impacting the excitation and evolution of the NGC 3393 ENLR in detail that complements and extends beyond the recent work of Koss et al. (2015).

Throughout this paper, we adopt concordant cosmological parameters $^{4}$ of $H_{0}=70 \mathrm{~km}^{-1} \mathrm{~s}^{-1} \mathrm{Mpc}^{-1}, \Omega_{m, 0}=0.3$, and

\footnotetext{
4 Distances are calculated according to http://www.astro.ucla.edu/ wright/ CosmoCalc.html.
} 
Table 1

Hubble Observation Properties

\begin{tabular}{|c|c|c|c|c|c|}
\hline Data Set & Obs Date & Exposure (s) & Instrument & Filter & Note \\
\hline IBIG06050 & 2011 Nov 11 & 2040 & WFC3/UVIS & F814W & I-band \\
\hline IBLY01011 & 2011 May 16 & 566 & WFC3/UVIS & FQ508N & [O III $]$ \\
\hline IBLY01021 & 2011 May 17 & 466 & WFC3/UVIS & F665N & $\mathrm{H} \alpha+[\mathrm{N}$ II $]$ \\
\hline IBLY01GWQ & 2011 May 17 & 208 & WFC3/UVIS & F547M & line continuum \\
\hline IBLY01GXQ & 2011 May 17 & 208 & WFC3/UVIS & F621M & line continuum \\
\hline IBLY01GYQ & 2011 May 17 & 314 & WFC3/UVIS & F673N & [S II $]$ \\
\hline
\end{tabular}

Note. IBIG exposures are from program 12185 (PI: Greene). IBLY exposures are from CHEERS, program 12365 (PI: Wang).

$\Omega_{\Lambda, 0}=0.7$. All coordinates are J2000. For distances, we use $D=53 \mathrm{Mpc}$, such that NGC 3393 presents an angular scale $256 \mathrm{pc} \operatorname{arcsec}^{-1}$.

\section{OBSERVATIONS AND DATA}

\subsection{HST Observations}

NGC 3393 has been observed extensively by HST over its mission, including WFPC1, WFPC2, FOS, STIS, ACS, and NICMOS. For this investigation, we mainly use observations taken for CHEERS (Program 12365). CHEERS observations were taken using the Wide Field Camera 3 (WFC3) instrument in a subarray configuration of the UVIS channel on 2011 May 16 and 17 using the FQ508N, F665N, F547M, F621M, and F673N filters. We also used observations taken with the F814W filter on 2011 November 11 (Program 12185, PI: Greene). These short observations lasted 566, 466, 208, 208, 314 , and $2040 \mathrm{~s}$, respectively, and are listed in Table 1 along with relevant bandpasses.

At the redshift of NGC 3393, our narrow filter observations cover $\sim 42 \AA$ (observer frame) for each of three bands, with FQ508N, F665N, and F673N covering [O III] $\lambda 5007$, $\mathrm{H} \alpha \lambda 6563$ with [N II] $\lambda \lambda 6548,6584$, and the [S II] $\lambda \lambda 6716$, 6731 doublet, respectively. We measure the the [O III] continuum using the F547M band, and the $\mathrm{H} \alpha+[\mathrm{N}$ II] and [S II] continuum from F621M. We expect all of the line and continuum bands to be free of contamination from other significant emission lines.

\subsection{Data Reduction}

We used the standard HST data processing package, DrizzlePac (Gonzaga et al. 2012), to process HST images with Pyraf. This software was installed with version 1.5.1 of Ureka. ${ }^{5}$ Cosmic rays and bright sources can leave faint residual charge trails in the direction of readout. Software is available for for WFC3 CTE correction ${ }^{6}$, but is not capable of handling the subarray configuration of most of our images and is therefore not used here. The most and longest observations are taken using the F814W filter with correspondingly better cosmic ray removal and catalog source significance. We therefore use the F814W images for astrometric reference. In each band, we reject cosmic rays using AstroDrizzle. We match subexposure astrometry using DrizzlePac tools TweakReg and TweakBack, and use AstroDrizzle again to produce a combined band image, varying the source catalog and cosmic ray criteria as necessary. We match the astrometry in different

\footnotetext{
http://ssb.stsci.edu/ureka/

6 http://www.stsci.edu/hst/wfc3/ins_performance/CTE/
}

bands to the final F814W images using TweakReg and TweakBack. We then match the pixel sampling to the native pixel scale $(\sim 0$ !' 04 width) and the orientation of the F814W images using AstroDrizzle.

Observations with filters F547M, F621M, and F673N have only single exposures, which eliminates the possibility of cosmic ray removal by direct comparison within a single filter band. We therefore use the final F814W image as a reference for cosmic ray removal in F547M and F621M. We use the F665N image as a reference for cosmic ray removal in the F673N band. Differences in surface brightness gradients when comparing images in different bands can create false cosmic ray detections for sufficiently aggressive cosmic ray removal criteria. We therefore use criteria which leave a number of cosmic rays and remove them manually with the Pyraf tool imedit. We also remove several cosmic rays from the FQ508N image using imedit.

Background determination is complicated by the large size of NGC 3393 relative to the fields of view of the WFC3 observations. This can cause background over-subtraction in the AstroDrizzle median sigma-clipping routine. The darkest continuum regions, for example, exhibit some flux when viewed at $3.6 \mu \mathrm{m}$ with Spitzer (Program 100098; PI: Stern). We therefore subtract a background component which matches the spatial gradients (in regions dominated by old stellar populations) seen in Spitzer. The resulting background is consistent with median values of the darkest regions to $\lesssim 10 \%$ for all images, and is less than the uncertainty in the observed continuum. Where the line flux $\geqslant 3 \sigma$, this background is no more than a few percent of line flux.

\subsection{Narrowline Mapping}

We produced emission line maps of [O III] $\lambda 5007, \mathrm{H} \alpha \lambda 6563$ and [S II] $\lambda \lambda 6716,6731$ by rescaling and subtracting the $H S T$ continuum bands from the corresponding narrowline filter observations. The CIAO tool dmimgcalc ${ }^{7}$ is capable of generic numerical manipulations of FITS image files and was used for this purpose. We rescaled these images according to the WFC3 absolute flux calibration keyword PHOTFLAM and by pixel size in $\operatorname{arcsec}^{2}$ to have images in units of erg cm ${ }^{-2} \mathrm{~s}^{-1} \AA^{-1} \operatorname{arcsec}^{-2}$. We adjusted the scaling of the continuum prior to subtraction, such that regions with both high-signal-to-noise ratio continuum and negligible expected line emission (i.e., outside the ionization cones) produce median continuum-subtracted flux values consistent with zero.

To map [O III] $\lambda 5007$, we simply rescaled the F547M image to match FQ508N regions outside the ionization cones and

7 http://cxc.harvard.edu/ciao/ahelp/dmimgcalc.html 

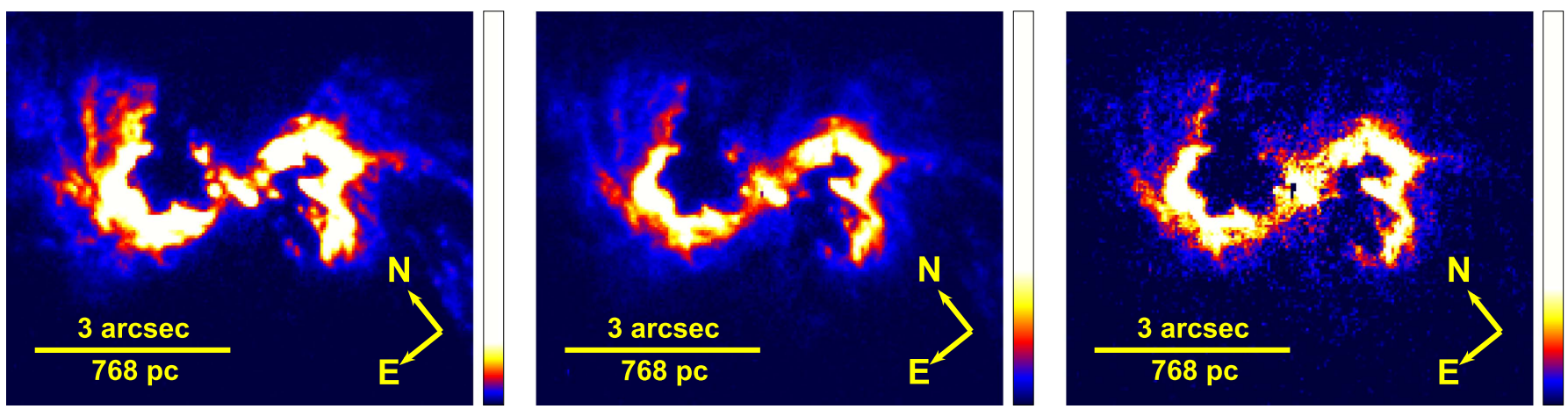

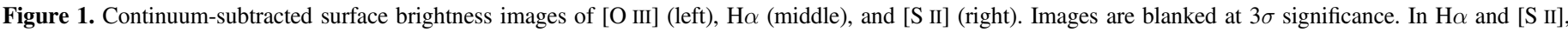
there is a flaw near the nucleus a few pixels in size due to poor cosmic ray subtraction in the continuum band.

inner $\sim 2 \mathrm{kpc}$, then subtracted the result from FQ508N, assuming excess flux is due solely to [O III] $\lambda 5007 . \mathrm{H} \alpha \lambda 6563$ and $[\mathrm{S}$ II] $\lambda \lambda 6716,6731$ require more complicated modeling due to variation of filter transmission and contamination by $[\mathrm{N}$ II] $\lambda \lambda 6548,6584$. We subtracted the F621M continuum similarly from F665N and F673N. We then modeled the respective contributions of different lines' emission to the total count rate in filters using pysynphot from Ureka to simulate the expected count rates in F665N and F673N. According to Cooke et al. (2000), [N II] varies little with radius on spatial scales of $\lesssim 1^{\prime \prime}$. We therefore assume Gaussian emission profiles with FWHM $336 \mathrm{~km} \mathrm{~s}^{-1}$ and line strengths according to Table 7 in Cooke et al. (2000), taken from ground-based spectroscopy.

To correct for line filling from $\mathrm{H} \alpha$ absorption in the host galaxy starlight, we use methods based on Keel (1983). We extrapolate the relative continuum strength at 5500 and $6700 \AA$ from F547M and F621M and use pysynphot and dmimgcalc to calculate fluxes. We correct $\mathrm{H} \alpha$ for continuum absorption from the expected equivalent width based on the continuum slope as per Keel (1983). We use the CIAO tool dmimgthresh and Pyraf to limit the range of $\mathrm{H} \alpha$ absorption equivalent width to between 0 and $2.6 \AA$, which is the largest value considered by Keel (1983). The brightest features in the nuclear ENLR do exceed 2.6 $\AA$, suggesting estimates of the stellar continuum in the arms for F547M and F621M are strongly affected by local gas, possibly due to [O I] $\lambda \lambda 6000,6300$ emission falling within F621M. Using the Keel (1983) relationship between color and absorption, we find $\mathrm{H} \alpha$ filling to be a minor effect, $\lesssim$ few $\%$. The final line continuum-subtracted line maps are presented in Figure 1. These images are blanked at $3 \sigma$ (as per Cresci et al. 2015) to emphasize regions that are sufficiently bright for line ratio mapping at maximum resolution without additional processing.

\section{BPT MAPPING}

We use the formalism of Kewley et al. (2006) to produce resolved BPT maps of the inner $\sim \mathrm{kpc}$ radius region $\left(\sim 4^{\prime \prime}\right)$ of NGC 3393. To do this, we divide pairs of emission line maps from Section 2.3 and Figure 1 to create ratio maps of [S II]/H $\alpha$ and $[\mathrm{O} \mathrm{III}] / \mathrm{H} \beta$ at the native WFC3 pixel size. A value of $\mathrm{H} \alpha \sim 3 \times \mathrm{H} \beta$ has long been shown to be typical of AGN NLRs (Halpern \& Steiner 1983; Gaskell \& Ferland 1984) and is consistent with the Cooke et al. (2000) value for NGC 3393. We therefore use $\mathrm{H} \alpha$ as a proxy for $\mathrm{H} \beta$ according to this ratio.

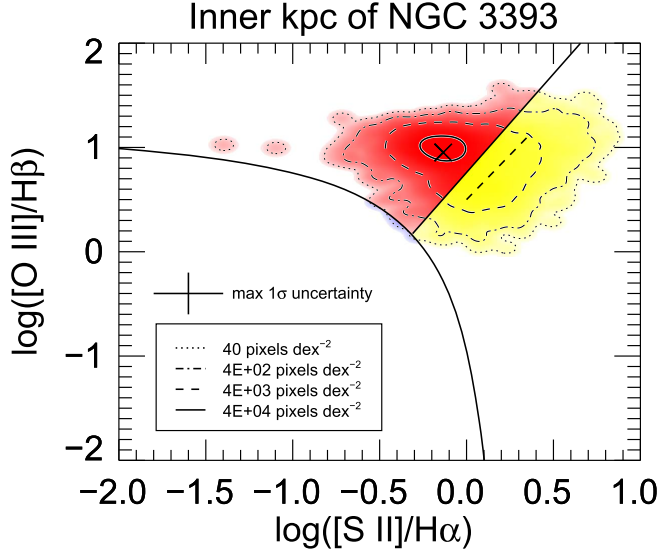

Figure 2. BPT Diagram of the inner kpc radius region of NGC 3393, covering a $\sim 7^{\prime \prime} \times 7^{\prime \prime}$ NE-SW region centered on the nucleus and ionization cones. As in Figure 3, red corresponds to Seyfert-like activity (top left), yellow corresponds to LINER-like activity (right), and blue pixels have line ratios typical of $\mathrm{H}$ II regions (bottom left). Solid black lines mark the boundaries of these regions according to Kewley et al. (2006). Contours and intensity of color indicate phase space density, in terms of number of WFC3 pixels per square dex in the BPT diagram where $\mathrm{S} / \mathrm{N} \geqslant 3 \sigma$. Most $\mathrm{H}$ II-like pixels are excluded by this criterion. An "X" marks the integrated fluxes where $r<2$ " from the nucleus. The cross indicates the $1 \sigma$ uncertainty of any pixel which is only detected at $3 \sigma$ in all lines. A dashed line indicates the elongated parameter space overdensity, mentioned in the text but not visible in this color stretch.

In Figure 2, we plot a resolved BPT diagram of $\log ([\mathrm{O} \mathrm{III}] / \mathrm{H} \beta)$ versus $\log ([\mathrm{S} \mathrm{II}] / \mathrm{H} \alpha)$, such that each 0 ! $04 \times 0$ ! 04 WFC3 pixel $(10 \mathrm{pc} \times 10 \mathrm{pc})$ represents one data point on the BPT diagram. The extraction region covers a $\sim 7^{\prime \prime} \times 7^{\prime \prime}$ region aligned NE to SW which includes the nucleus (center) and the ionization cones. We use $3 \sigma$ blanking as a requirement for emission line significance (as in Cresci et al. 2015).

Figure 2 shows all the $3 \sigma$-blanked image pixels in log $([\mathrm{O} \mathrm{III}] / \mathrm{H} \beta)$ versus $\log ([\mathrm{S} \mathrm{II}] / \mathrm{H} \alpha)$ space. Contours of pixel density are shown. The cross marks the peak, which lies in the Seyfert region, in agreement with line ratios from the integrated nuclear region at $r<2^{\prime \prime}$. A secondary elongated structure is in the LINER region (not visible in this color stretch) oriented along the LINER/Seyfert divide, such that $[\mathrm{O}$ III $] / \mathrm{H} \beta$ increases along with $[\mathrm{S} \mathrm{II}] / \mathrm{H} \alpha$.

We can examine the location of different emission line excitation classes by displaying an image of the circumnuclear region such that each WFC3 pixel is color-coded according to its position on the BPT diagram. Figure 3 shows the BPT 


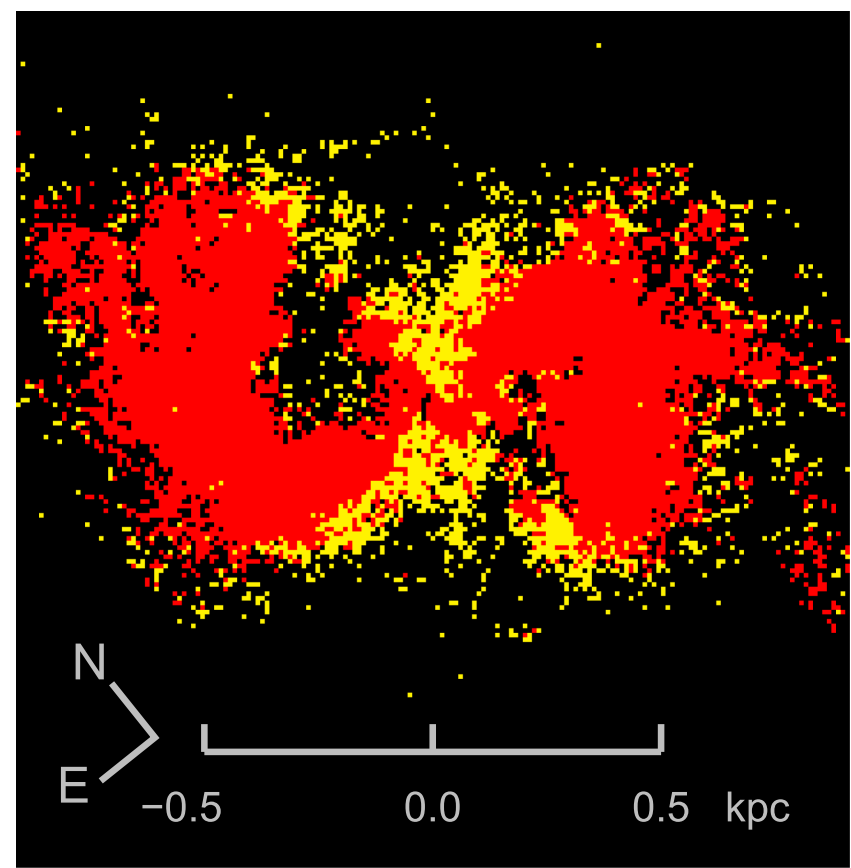

Figure 3. Resolved BPT image of the inner kpc radius region of NGC 3393, covering a $\sim 7^{\prime \prime} \times 7^{\prime \prime} \mathrm{NE}-\mathrm{SW}$ region $\left(7^{\prime \prime} \sim 1.8 \mathrm{kpc}\right)$ centered on the nucleus and ionization cones. Each WFC3 pixel has been colored according to its position on the BPT diagram in Figure 2 relative to its $[\mathrm{S} \mathrm{II}] / \mathrm{H} \alpha$ and $[\mathrm{O}$ III] $/ \mathrm{H} \beta$ ratios. Red corresponds to Seyfert-like activity and yellow corresponds to LINER-like activity. Black pixels have at least one line where $F_{\text {line }} \leqslant 3 \sigma$ in this region. Blue pixels represent line ratios typical of $\mathrm{H}$ II regions and are entirely excluded here due to weak line significance.

image of NGC 3393. Red indicates Seyfert-type activity, yellow is LINER-like, and blue is typical of star-forming regions. Pixels without at least $\sim 3 \sigma$ detection are excluded (black).

We immediately see several important features of the morphology:

(1) Within $r \sim 3^{\prime \prime}(\sim 770 \mathrm{pc})$ of the nucleus, the ionization cones are dominated by an S-shaped structure filled with Seyfert-type emission (red).

(2) Narrowline emission within $r \sim 3^{\prime \prime}$ but outside the cones is predominantly unclassified due to weak line significance (particularly in [S II]). Our $3 \sigma$ blanking criterion (black in Figure 3) selects against low-emission regions, which may also select against H II-like regions at the lines' limiting fluxes (Figure 3; blue).

(3) The interface between these two spatial regions is an open figure 8 of LINER-type emission (yellow in Figure 3). This interface is thin, $\lesssim 100 \mathrm{pc}(\lesssim 0$ !" 4$)$ in projection. This LINER-like "cocoon" is a newly identified structure in this galaxy.

\section{DISCUSSION}

Narrowline imaging of [S II] and reasonable assumptions about $\mathrm{H} \alpha / \mathrm{H} \beta$ and $\mathrm{H} \alpha /[\mathrm{NII}]$ (described in Section 3) have allowed us to study the excitation of the ENLR on scales of $\sim 10$ pc by placing it on the BPT diagram. Figure 3 shows a very clean morphology for the inner $\sim 770 \mathrm{pc}\left(\sim 3^{\prime \prime}\right)$.

(1) Because the ionization cone interiors are almost entirely comprised of Seyfert-like emission, we may simply suppose their origin is predominantly by photoionization. However, if the $\mathrm{S}$-shape is formed by fast shocks with photoionized precursors (as considered by Cooke et al. 2000), shock velocities of $\gtrsim 500 \mathrm{~km} \mathrm{~s}^{-1}$ can produce photoionization-like BPT positions (Allen et al. 2008).

(2) The cross-cone region emission becomes too faint to classify $\sim 300 \mathrm{pc}$ from the nucleus. This limits our ability to measure the full extent of the LINER cocoon, which may be much thicker than $\sim 100 \mathrm{pc}$. Deeper observations are necessary to show if the cocoon is bounded by a lower-ionization H IIlike region (as in Cresci et al. 2015).

(3) The LINER-like "cocoon" may be due to shock production (Heckman 1980; Allen et al. 2008) or consist of regions with large local gas densities relative to the AGN ionizing flux (hence their low ionization) or they are regions receiving diluted radiation due to filtering of the nuclear radiation (Kraemer et al. 2008; Heckman \& Best 2014) due to shielding by the the edge of the torus or by a hollow biconical AGN wind, each of which may create a zone of lower ionization.

Kraemer et al. (2008) distinguish two ionization states in the base of the NGC 4151 wind out to $\sim 70$ pc using [O III]/[O II]. Using only two lines, however, they can only distinguish high and low ionization states. Our analysis is consistent with that picture, such that the addition of a third line gives a cleaner classification. We resolve larger-scale structure in finer detail, however $(10 \mathrm{pc}$ pixels at all scales here, versus wedges of 11-44 pc angular extent at $r \sim 30-130 \mathrm{pc}$ in NGC 4151), allowing us to see the thin LINER cocoon. Even with an additional diagnostic line for BPT analysis, the $20^{\circ}$ azimuthal bins used by Kraemer et al. (2008) would be too large to resolve this LINER-like region, and each pixel in our map is comparable $(\sim 10 \mathrm{pc})$ to one of their radial bin steps.

The LINER-like emission is located in a "cocoon" surrounding the Seyfert emission regions. Shocks resulting from gas expanding outward from the ionization cone with $v \gtrsim 200 \mathrm{~km} \mathrm{~s}^{-1}$ could explain these regions as the result of the AGN wind pushing into the surrounding ISM.

Whether shocks play a significant role, the origin of the LINER-like cocoon could be analogous to the "Fermi bubbles" in our own Milky Way galaxy, assuming those bubbles were driven by an AGN wind in the recent past ( $\mathrm{Su}$ et al. 2010).

The detailed morphology of the ENLR suggests that different morphologies of obscuration and gas distribution may affect the classification of a galaxy taken as a whole, e.g., at high redshift or in a SDSS $3^{\prime \prime}$ fiber Kewley et al. (2013). Modeling is necessary to fully investigate the effects of plausible geometries, but that is beyond the scope of this paper.

\section{CONCLUSIONS}

Through use of narrowband HST images, we produced a resolved BPT diagram and BPT map of a $\sim 7^{\prime \prime} \times 7^{\prime \prime}(\sim 1.8 \mathrm{kpc}$ square) region covering the nucleus and inner ionization cones of NGC 3393. We showed that the regions within the bicones are typically consistent with Seyfert galaxies and consistent with previous classification of the galaxy based on the nucleus alone, while the line emission outside these regions has line surface brightness too low to categorize at full resolution. The surprise is that we find a thin LINER-like "cocoon" bounding the AGN-like region. Deeper observations (such as with HST and James Webb Space Telescope) are necessary to measure the cocoon's full extent and answer the question: is the cocoon as thin as it seems? 
Our ability to distinguish these different regions is limited by the availability of different diagnostic lines and by the resulting degeneracies between the models within the observed parameters.

The BPT maps are consistent with the LINER cocoon at the edge of the bicone being shielded by the torus or AGN wind, or with shocks.

Further investigation of these mechanisms is therefore necessary. In the next paper of this series (W. P. Maksym et al. 2016, in preparation), we will investigate the limits of the broad set of available observational data for NGC 3393, including a more detailed study of the optical morphology in conjunction with high-resolution radio and X-ray data. That work will provide the basis for a detailed study of Chandra emission line imaging data. Given the role that optical narrowline ratios play in understanding the ENLR, we expect X-ray mapping of important diagnostic lines like Ne IX, O VII and $\mathrm{O}$ VIII to provide a similarly important, complementary, role in understanding ENLR processes in AGNs, as demonstrated by Wang et al. (2011a, 2011b) and Paggi et al. (2012). A deeper understanding of the morphology on sub-kpc scales will better inform methods of X-ray spectral extraction, which is critical given the limitations due to low photon counting rates in the high-resolution regime of X-ray imaging.

W.P.M. acknowledges support from Chandra grants GO415107X, GO5-16099X, and GO2-13127X, and Hubble grant GO-13741.002-A, and also thanks Henrique Schmitt for helpful discussions. J.W. acknowledges support from the NSFC grants 11473021 and 11522323, and the Fundamental Research Funds for the Central Universities under grant 20720160023. We acknowledge partial support by NASA contract NAS8-03060 (CXC). STSDAS and PyRAF are products of the Space Telescope Science Institute, which is operated by AURA for NASA. We thank the anonymous referee for comments that improved the quality of the paper. M. E. and G.F. thank the Aspen Center for Physics, funded by NSF grant \#1066293 and for their hospitality while this paper was completed.

Facilities: HST, Spitzer.

\section{REFERENCES}

Allen, M. G., Groves, B. A., Dopita, M. A., Sutherland, R. S., \& Kewley, L. J. 2008, ApJS, 178, 20

Baldwin, J. A., Phillips, M. M., \& Terlevich, R. 1981, PASP, 93, 5

Barbosa, F. K. B., Storchi-Bergmann, T., McGregor, P., Vale, T. B., \& Rogemar Riffel, A. 2014, MNRAS, 445, 2353

Baumgartner, W. H., Tueller, J., Markwardt, C. B., et al. 2013, ApJS, 207, 19

Cooke, A. J., Baldwin, J. A., Ferland, G. J., Netzer, H., \& Wilson, A. S. 2000, ApJS, 129, 517

Cresci, G., et al. 2015, A\&A, 582, A63

Davies, R. L., Dopita, M. A., Kewley, L. J., et al. 2016, ApJ, 824, 50

de Vaucouleurs, G., de Vaucouleurs, A., Corwin, H. G., Jr., et al. 1991, Third Reference Catalogue of Bright Galaxies, Vol. I, II, III (New York, NY: Springer)

Fabian, A. C. 2012, ARA\&A, 50, 455

Gaskell, C. M., \& Ferland, G. J. 1984, PASP, 96, 393

Gaskin, J. A., Weisskopf, M. C., Vikhlinin, A., et al. 2015, Proc. SPIE, 9601, $96010 \mathrm{~J}$

Gonzaga, S., Hack, W., Fruchter, A., \& Mack, J. 2012, The DrizzlePac Handbook (Baltimore, MD: STScI)

Halpern, J. P., \& Steiner, J. E. 1983, ApJL, 269, L37

Heckman, T. M. 1980, A\&A, 87, 152

Heckman, T. M., \& Best, P. N. 2014, ARA\&A, 52, 589

Ho, I.-T., Kewley, L. J., Dopita, M. A., et al. 2014, MNRAS, 444, 3894

Ho, L. C. 2008, ARA\&A, 46, 475

Keel, W. C. 1983, ApJ, 269, 466

Kewley, L. J., Dopita, M. A., Leitherer, C., et al. 2013, ApJ, 774, 100

Kewley, L. J., Dopita, M. A., Sutherland, R. S., Heisler, C. A., \& Trevena, J. 2001, ApJ, 556, 121

Kewley, L. J., Groves, B., Kauffmann, G., \& Heckman, T. 2006, MNRAS, 372,961

Kondratko, P. T., Greenhill, L. J., \& Moran, J. M. 2008, ApJ, 678, 87

Koss, M. J., et al. 2015, ApJ, 807, 149

Kraemer, S. B., Schmitt, H. R., \& Crenshaw, D. M. 2008, ApJ, 679, 1128

Levenson, N. A., Heckman, T. M., Krolik, J. H., Weaver, K. A., \& Życki, P. T. 2006, ApJ, 648, 111

Maiolino, R., Salvati, M., Bassani, L., et al. 1998, A\&A, 338, 781

Nisbet, D. M., \& Best, P. N. 2016, MNRAS, 455, 2551

Paggi, A., Wang, J., Fabbiano, G., Elvis, M., \& Karovska, M. 2012, ApJ, 756,39

Silk, J., \& Rees, M. J. 1998, A\&A, 331, L1

Storchi-Bergmann, T., Lopes, R. D. S., McGregor, P. J., et al. 2010, MNRAS, 402, 819

Su, M., Slatyer, T. R., \& Finkbeiner, D. P. 2010, ApJ, 724, 1044

Theureau, G., Bottinelli, L., Coudreau-Durand, N., et al. 1998, A\&AS, 130,333

Wang, J., Fabbiano, G., Elvis, M., et al. 2011a, ApJ, 736, 62

Wang, J., et al. 2011b, ApJ, 742, 23

Winter, L. M., Veilleux, S., McKernan, B., \& Kallman, T. R. 2012, ApJ, 745,107 